\title{
Resultado funcional do procedimento de tenodese extra-articular lateral (TEL) como complemento à reconstrução do ligamento cruzado anterior: Uma metanálise*
}

\section{Functional Outcome of Lateral Extraarticular Tenodesis Procedure in Addition to Anterior Cruciate Ligament Reconstruction: A Metaanalysis}

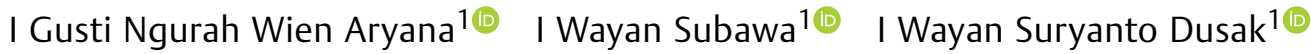 \\ Cokorda Gde Oka Dharmayuda1[i] Hans Kristian Nugraha ${ }^{1}$ Maria Florencia Deslivia1이 \\ ${ }^{1}$ Departamento de Ortopedia e Traumatologia, Faculdade de Medicina \\ Udayana University, Sanglah General Hospital, Bali, Indonésia \\ Rev Bras Ortop 2022;57(1):33-40. \\ Endereço para correspondência I Gusti Ngurah Wien Aryana, MD, \\ RSUP Sanglah, Orthopaedi dan Traumatologi, Jl. Diponegoro, Dauh \\ Puri Klod, Kec. Denpasar Bar., Kota Denpasar, Bali, Denpasar, Bali \\ 80113, Indonésia (e-mail: wienaryana@unud.ac.id).
}

\section{Resumo}

Palavras-chave

- reconstrução do ligamento cruzado anterior

- lesões do ligamento cruzado anterior

- instabilidade articular

- tenodese
Objetivos O objetivo deste estudo é revisar e analisar sistematicamente o desfecho funcional do procedimento de tenodese extra-articular lateral (TEL) em complemento à reconstrução do ligamento cruzado anterior (RLCA) em de estudos com alto nível de evidências.

Métodos Realizamos a pesquisa bibliográfica para estudos clínicos comparando o método TEL como complemento à RCLA com a RLCA isolada. Os resultados principais foram a pontuação no Comitê Internacional de Documentação de Joelho (IKDC, na sigla em inglês), pontuação de Lysholm, e falhas no enxerto. Variáveis contínuas foram relatadas, como médias e intervalos de confiança (ICs) de $95 \%$.

Resultados Seis estudos clínicos com 1,049 pacientes foram incluídos na metanálise. O período de seguimento foi de, em média, 24 meses (intervalo de 6-63 meses). A adição do procedimento TEL à reconstrução do LCA resultou em melhor resultado funcional com base no escore IKDC $(p<0,05)$. A falha do enxerto foi menor no grupo RLCA mais TEL (16 dos 342 pacientes) em comparação com o grupo apenas RLCA (46 dos 341 pacientes) $(p<0,05)$.

Conclusão Há evidências de alto nível de que o procedimento TEL como complemento à RLCA é preferível em termos de resultado funcional e falha do enxerto.
Trabalho desenvolvido no Departamento de Ortopedia e Traumatologia, Faculdade de Medicina Udayana University, Sanglah General Hospital, Bali, Indonésia. recebido

19 de Fevereiro de 2021

aceito

15 de Junho de 2021

Publicado on-line

de Janeiro 21, 2022
DOI https://doi.org/

10.1055/s-0041-1736514. ISSN 0102-3616. (c) 2022. Sociedade Brasileira de Ortopedia e Traumatologia. All rights reserved.

This is an open access article published by Thieme under the terms of the Creative Commons Attribution-NonDerivative-NonCommercial-License, permitting copying and reproduction so long as the original work is given appropriate credit. Contents may not be used for commercial purposes, or adapted, remixed, transformed or built upon. (https://creativecommons.org/ licenses/by-nc-nd/4.0/)

Thieme Revinter Publicações Ltda., Rua do Matoso 170, Rio de Janeiro, RJ, CEP 20270-135, Brazil 


\author{
Abstract \\ Keywords \\ - anterior cruciate \\ ligament \\ reconstruction \\ - anterior cruciate \\ ligament injuries \\ - joint instability \\ - tenodesis
}

Objectives The aim of the present study is to systematically review and analyze the functional outcome of lateral extraarticular tenodesis (LET) procedure in addition to anterior cruciate ligament reconstruction (ACLR) in studies with a high level of evidence.

Methods We performed a literature search for clinical studies comparing the LET method as an augmentation to $A C L$ reconstruction with $A C L$ reconstruction alone. The primary outcomes were the International Knee Documentation Committee (IKDC) score, the Lysholm score, and graft failures. Continuous variables were reported as means and $95 \%$ confidence intervals (Cls).

Results Six clinical studies with 1,049 patients were included in the metaanalysis. The follow-up period was, in average, 24 months (range, 6-63 months). The addition of the LET procedure to ACLR results in better functional outcome based on the IKDC score $(p<0.05)$. Graft failure was found to be lower in the ACLR plus LET group (16 of 342 patients) compared with the ACLR-only group (46 of 341 patients) $(p<0.05)$.

Conclusion There is high-level evidence that LET procedure in addition to ACLR is preferable in terms of functional outcome and graft failure.

\section{Introdução}

Técnicas tradicionais de reconstrução do ligamento cruzado anterior (RLCA) de feixe único têm demonstrado fornecer bons resultados subjetivos; no entanto, vários estudos têm demonstrado que muitos pacientes continuam a ter complicações relacionadas ao procedimento. A falha da RLCA pode ser causada pela instabilidade rotacional anterolateral devido à inadequação do enxerto intra-articular para recriar uma cinemática normal do joelho. ${ }^{1}$ Uma das soluções propostas é utilizar técnicas reconstrutivas de tecido mole de base lateral, além da RLCA. Esta metanálise discutirá a tenodese extra-articular lateral (TEL) como a técnica de aumento para a R LCA.

A TEL não é um conceito novo. Foi originalmente usada para tratar o joelho com deficiência de LCA na ausência de técnicas de reconstrução intra-articular. O objetivo do procedimento é colocar uma contenção lateral do tecido mole a uma distância do pivô central do joelho, melhorando assim a vantagem mecânica para controlar a rotação ao tratar esse joelho. ${ }^{2}$ Juntamente com o uso generalizado da reconstrução intra-articular, em particular a introdução de técnicas artroscópicas, a TEL tornou-se menos conhecida, especialmente porque não havia vantagem adicional comprovada com sua aplicação. ${ }^{3}$

O paradigma mudou quando um foco significativo foi colocado sobre o complexo anterolateral (CAL). No lado lateral do joelho, há estruturas de tecidos moles que têm a função de prevenir a frouxidão rotatória anterolateral. O CAL inclui as bandas iliotibiais (BIs) superficial e profunda, a camada capsulo-óssea da BI, e um espessamento da cápsula lateral referida como o ligamento anterolateral (LAL). ${ }^{4} \mathrm{O} \mathrm{LAL}$ foi descrito por muitos autores como ou o ligamento capsular médio, a camada capsulo-óssea da BI, ou uma combinação de ambos. ${ }^{5}$ Estudos cadavéricos mostraram que, em conjunto com a RLCA, a TEL é uma excelente técnica cirúrgica para controlar a frouxidão rotatória anterolateral do joelho devido a lesão ou deficiência no CAL. ${ }^{6}$ Houve também revisões sistemáticas anteriores, e a metanálise relatou bons resultados a médio prazo, mesmo com um número limitado de pacientes. ${ }^{7-10}$

O objetivo deste estudo é reunir evidências dos mais recentes ensaios controlados randomizados (ECRs) em TEL sobre seu resultado funcional e complicação como um complemento ao procedimento de reconstrução da LCA.

\section{Materiais e Métodos}

Esta revisão sistemática e metanálise foi realizada de acordo com a diretriz do PRISMA.

\section{Critérios de Estratégia de Pesquisa e Seleção}

Realizamos pesquisa da literatura usando os bancos de dados da PubMed/MEDLINE e Google Scholar. Não houve limitação quanto à data de publicação. A língua foi limitada ao inglês. Os termos da pesquisa incluíram, mas não se limitaram a: "ligamento extra-articular lateral, tenodese, reconstrução do ligamento anterolateral e reconstrução do ligamento cruzado anterior.

Todos os tipos de ensaios clínicos publicados como artigo completo foram incluídos neste estudo. Os artigos foram selecionados com base nos critérios de inclusão e exclusão segundo do modelo população, intervenção, comparação, resultado (PICO, na sigla em inglês), conforme descrito na -Tabela 1.

\section{Extração de Dados}

Os artigos foram selecionados por uma equipe de pesquisa, com cada artigo tendo sido examinado independentemente 
Tabela 1 Critérios de inclusão e exclusão com base no PICO

\begin{tabular}{|c|c|c|}
\hline & Critérios de inclusão & Critérios de exclusão \\
\hline População & $\begin{array}{l}\text { Pacientes com idade igual ou } \geq 18 \text { anos com ruptura } \\
\text { primária da LCA e planejavam passar pela reconstrução } \\
\text { da LCA com artroscopia. }\end{array}$ & $\begin{array}{l}\text { - Estudos em animais. } \\
\text { - Casos de revisão da reconstrução da LCA. } \\
\text { - Reconstrução concomitante de LCP ou menisco. } \\
\text { - Condição congênita subjacente ou neoplasia. }\end{array}$ \\
\hline Intervenção & $\begin{array}{l}\text { Reconstrução da LCA com tenodese extra-articular } \\
\text { lateral. }\end{array}$ & $\begin{array}{l}\text { - Reconstrução da LCA com reconstrução do LAL. } \\
\text { - Tratamento farmacológico. } \\
\text { - Tratamento nutricional. } \\
\text { - Fisioterapia ou reabilitação que funcione sozinha. }\end{array}$ \\
\hline Controle & Somente reconstrução da LCA. & \\
\hline Resultado & $\begin{array}{l}\text { Medidas de resultado primário } \\
\text { Os resultados clínicos, incluindo a pontuação do IKDC, } \\
\text { a EAV para dor e o SF-36 para qualidade de vida são os } \\
\text { resultados primários. A diferença média será relatada } \\
\text { com intervalos de confiança de } 95 \% \text {. } \\
\text { Medidas de desfecho secundário } \\
\text { Resultados adicionais de interesse incluem } \\
\text { complicações. Os desfechos dicotômicos serão } \\
\text { medidos utilizando-se razões de risco com intervalos de } \\
\text { confiança de } 95 \% \text {. }\end{array}$ & $\begin{array}{l}\text { - O estudo está em andamento e nenhum resultado } \\
\text { foi relatado } \\
\text { - Medidas de desfecho não relatadas na conclusão }\end{array}$ \\
\hline
\end{tabular}

Abreviaturas: EAV, escala analógica visual; IKDC, Comitê Internacional de Documentação de Joelho; LAL, ligamento anterolateral; LCA, ligamento cruzado anterior; LCP, ligamento cruzado posterior.

por dois membros da equipe (H.N. e M.F.D.). As divergências entre os revisores sobre a inclusão ou exclusão de um estudo foram resolvidas por consenso e, se necessário, consulta com um terceiro revisor.

Os dados foram coletados de cada artigo por dois revisores independentes, com divergências resolvidas por consenso e, se necessário, consulta com um terceiro revisor. Os dados foram registrados em um formulário desenvolvido a priori.

As variáveis abstratas incluíram idade do paciente, gênero, tamanho da amostra, acompanhamento médio, técnica de aumento de LAL, enxerto de aumento do LAL, técnica de RLCA, enxerto RLCA, escore Comitê Internacional de Documentação de Joelho (IKDC, na sigla em inglês), escore de Lysholm e complicações (por exemplo, falha no enxerto).

\section{Avaliação da Qualidade}

Os ensaios clínicos incluídos foram avaliados em termos de qualidade por dois revisores independentes com base em 13 itens da diretriz de método atualizado para revisões sistemáticas de 2015 do Cochrane Back and Neck Group. ${ }^{11}$ Foram avaliados os seguintes domínios para cada estudo incluído: randomização (viés de seleção), ocultação de alocação (viés de seleção), cegamento dos participantes e pessoal (viés de desempenho), cegamento da avaliação de resultados (viés de detecção), taxa de abandono (viés de atrito), todos os participantes analisados em grupo próprio (viés de atrito), viés de relatório seletivo (viés de relato), linha de base semelhante em relação aos indicadores prognósticos mais importantes (viés de seleção), cointervenções (viés de desempenho), conformidade aceitável em todos os grupos (viés de desempenho), e outras fontes de viés (patrocínio da indústria, por exemplo). As divergências durante a avaliação da qualidade foram resolvidas por meio de discussão e consenso e, se necessário, consulta com um terceiro revisor.

\section{Síntese de Dados}

As diferenças médias na pontuação do IKDC, pontuação de Lysholm, e falhas no enxerto foram os resultados primários investigados. Variáveis contínuas foram relatadas, tais como médias e intervalos de confiança (ICs) de 95\%. Os desfechos dicotômicos foram medidos utilizando-se razões de risco com ICs de $95 \%$. A heterogeneidade foi determinada por estimar a proporção de inconsistências entre estudos por diferenças reais, em vez de diferenças por erro aleatório ou por acaso, utilizando-se a estatística I, com valores de $25 \%$, $50 \%$ e $75 \%$ indicando baixos, moderados e altos graus de heterogeneidade, respectivamente. A análise estatística foi realizada utilizando-se RevMan versão 5.4 (Nordic Cochrane Center, Copenhague, Dinamarca). Um valor de $p<0,05$ foi considerado significativo.

\section{Resultados}

\section{Características de Pesquisa e Estudo da Literatura}

A pesquisa eletrônica preliminar de todas as bases de dados resultou em 1.364 registros, que foram triados por duplicatas, período de publicação, metodologia de estudo (apenas ECRs foram incluídos) e linguagem. Os artigos restantes foram posteriormente estudados por dois pesquisadores independentes com base no texto completo extraído utilizando um formulário desenvolvido a priori. Este processo seletivo rendeu 6 artigos finais a serem incluídos na metanálise, com o fluxo de processo seletivo retratado na - Fig. 1. Todos os artigos apresentaram alta qualidade de evidência, exceto aquele que tinha qualidade moderada ${ }^{12}$ (- Tabela 2 ). 


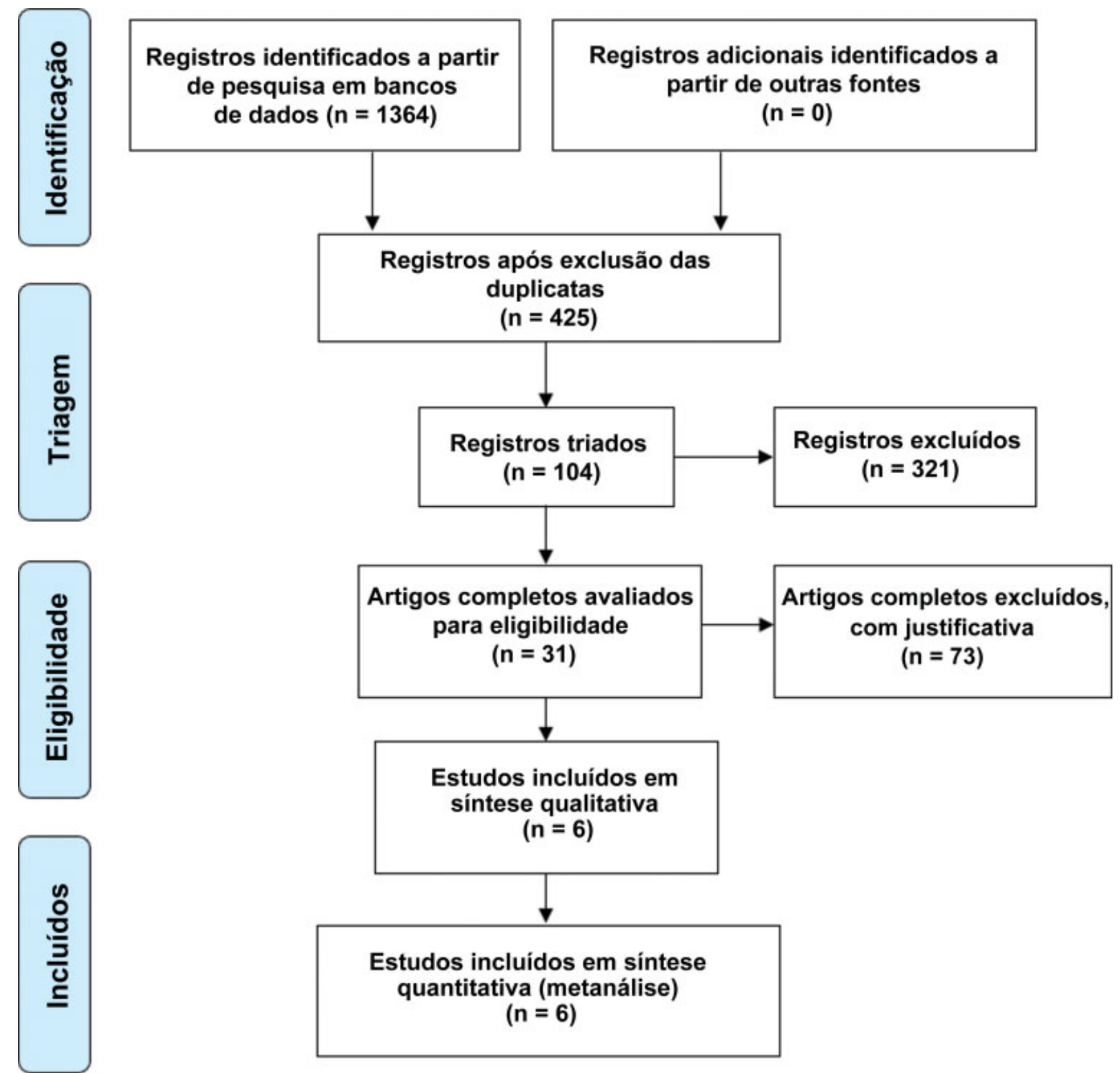

Fig. 1 Gráfico de fluxo mostrando seleção de artigos.

\section{Características da Linha de Base}

No estudo, foram investigados 1.049 pacientes. A lista completa das características da linha de base pode ser vista na - Tabela 3. Dos seis estudos, quatro utilizaram a técnica Lemaire ${ }^{13}$ para a tenodese extra-articular lateral, enquanto dois estudos utilizaram técnicas descritas uma por MacIntosh ${ }^{14}$ e uma por Christel. ${ }^{15}$ A BI foi utilizada para a tenodese em seis estudos, e o enxerto grácil foi utilizado em dois estudos. A técnica de reconstrução do LCA foi variada em todos os estudos, com três deles utilizando enxerto de LCA a partir do tendão isquiotibial (grácil e semitendíneo) e três utilizando enxerto ósseo do tendão patelar ósseo. 0 período de seguimento foi semelhante na maioria dos estudos, ou seja, em média 24 meses (intervalo, 6 a 63 meses), enquanto dois estudos tiveram acompanhamento de longo prazo, ou seja, mais de 10 anos de seguimento.

\section{Resultado Funcional}

Havia uma grande variedade de parâmetros clínicos que poderiam ser utilizados para avaliar o resultado de procedimentos no joelho. A lista completa do resultado funcional da reconstrução do TEL no LCA pode ser vista na - Tabela 4. O mais utilizado é o escore do IKDC, com três estudos utilizando este parâmetro ${ }^{1,16,17}$ favorecendo a RLCA juntamente com o procedimento TEL e um estudo resultando em diferença insignificante entre os dois procedimentos. ${ }^{18} \mathrm{~A}$ adição do procedimento TEL à RLCA resulta em diferença significativa do resultado funcional com base no escore IKDC $(p<0,05)$. A diferença média foi de $-0,71$ (IC95\%, -0,84--0,58). (-Figs. 2 e 3) Dois estudos confirmaram melhor pontuação de Lysholm com adição do procedimento TEL. ${ }^{12,19}$ 
TEL como complemento à reconstrução do ligamento cruzado anterior Aryana et al. 37

\begin{tabular}{|c|c|c|c|c|c|c|}
\hline 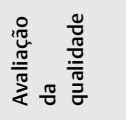 & 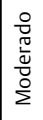 & $\frac{8}{4}$ & $\frac{9}{4}$ & $\frac{9}{4}$ & $\frac{9}{4}$ & $\frac{8}{4}$ \\
\hline 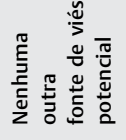 & E & $E$ & E & $\underline{E}$ & E & $E$ \\
\hline 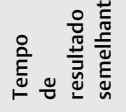 & E & E & E & E & E & $E$ \\
\hline 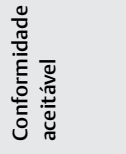 & 竞 & E & $\underline{E}$ & E & E & $E$ \\
\hline 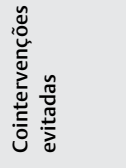 & E & $E$ & E & $\underline{E}$ & $\begin{array}{l}\text { 竘 } \\
\text { 殅 }\end{array}$ & $E$ \\
\hline 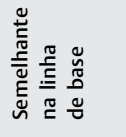 & E & $E$ & 㯊 & 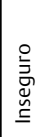 & 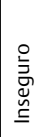 & $E$ \\
\hline 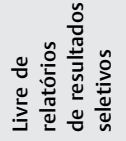 & 呞 & E & 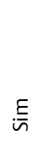 & 玹 & E & EE \\
\hline
\end{tabular}

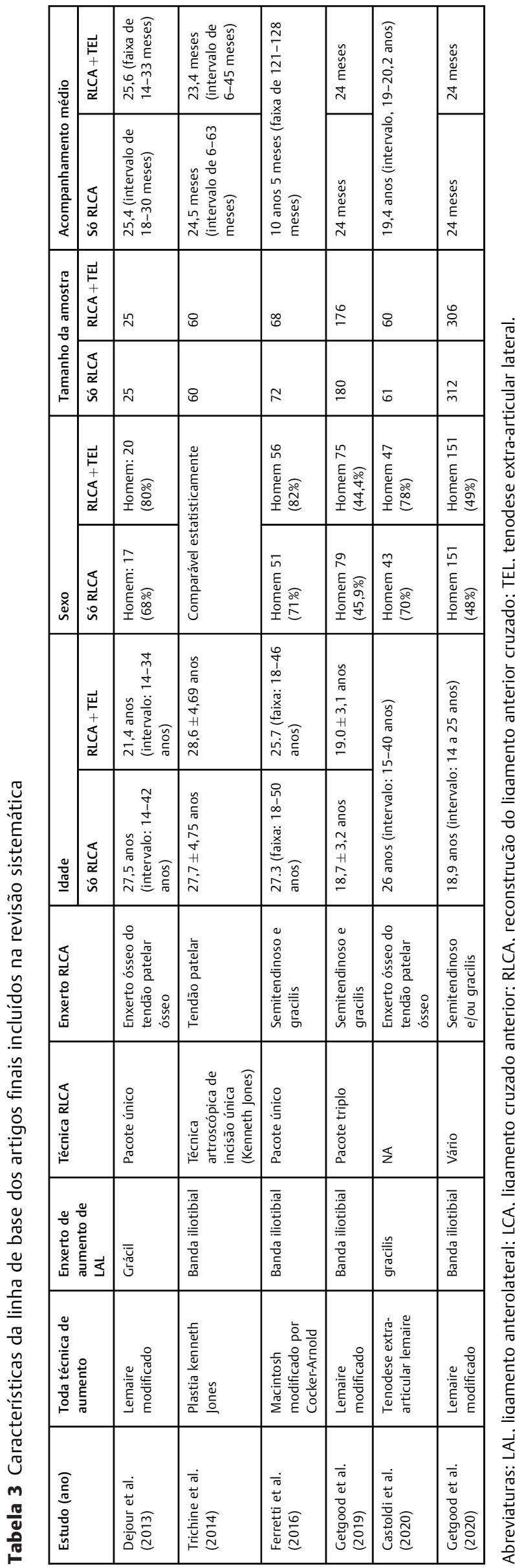


Tabela 4 Resultado funcional da tenodese extra-articular lateral na reconstrução do ligamento cruzado anterior de ensaios controlados randomizados recentes

\begin{tabular}{|l|l|l|l|l|l|l|}
\hline \multirow{2}{*}{ Estudo (ano) } & \multicolumn{2}{l|}{ IKDC } & \multicolumn{2}{l|}{ Lysholm } & \multicolumn{2}{l|}{ Falha no enxerto (\%) } \\
\cline { 2 - 7 } & Só RLCA & RLCA + TEL & Só RLCA & RLCA + TEL & Só RLCA & RLCA + TEL \\
\hline Dejour et al. (2013) & 90,1 & 86 & NA & NA & NA & NA \\
\hline Trichineet al. (2014) & 92,1 & 88,2 & NA & NA & NA & NA \\
\hline Ferretti et al. (2016) & 93,77 (DP 6,63) & 96,19 (DP 3,3) & 95,46 (DP 5,68) & 96,24 (DP 3,5) & NA & NA \\
\hline Getgood et al.(2019) & NA & NA & NA & NA & $11,67 \%$ & $13,07 \%$ \\
\hline Castoldi et al. (2020) & $\begin{array}{l}81,1 \text { (Intervalo, } \\
42,5-100)\end{array}$ & $\begin{array}{l}82,4 \text { (Intervalo, } \\
55,2-100)\end{array}$ & $86,6(42-100)$ & $90,3(67-100)$ & $47,54 \%$ & $21,67 \%$ \\
\hline
\end{tabular}

Abreviaturas: IKDC, Comitê Internacional de Documentação de Joelho; LAL, ligamento anterolateral; LCA, ligamento cruzado anterior; RLCA, reconstrução do ligamento anterior cruzado; TEL, tenodese extra-articular lateral.

\begin{tabular}{|c|c|c|c|c|c|c|c|c|c|c|}
\hline \multirow[b]{2}{*}{ Estudo ou subgrupo } & \multicolumn{3}{|c|}{ Somente RLCA } & \multicolumn{3}{|c|}{ RLCA + TEL } & \multirow[b]{2}{*}{ Peso } & \multirow{2}{*}{$\begin{array}{l}\text { Diferença média } \\
\text { IV, Fixo, } 95 \% \text { IC }\end{array}$} & \multirow{2}{*}{\multicolumn{2}{|c|}{$\begin{array}{l}\text { Diferença média } \\
\text { IV, Fixo, } 95 \% \text { IC }\end{array}$}} \\
\hline & Média & DP & Total & Média & DP & Total & & & & \\
\hline Castoldi 2020 & 81,1 & 14,74 & 61 & 82,4 & 14,73 & 60 & $0,1 \%$ & $-1,30[-6,55,3,95]$ & & \\
\hline Ferretti 2016 & 93,77 & 6,63 & 72 & 96,19 & 3,3 & 68 & $0,5 \%$ & $-2,42[-4,14,-0,70]$ & & \\
\hline Getgood 2020 & 86,6 & 0,8 & 312 & 87,3 & 0,8 & 306 & $99,4 \%$ & $-0,70[-0,83,-0,57]$ & & \\
\hline Trichine 2014 & 86 & 54,7 & 60 & 87 & 54,7 & 60 & $0,0 \%$ & $-1,00[-20,57,18,57]$ & $\leftarrow$ & \\
\hline Total (95\% IC) & & & 505 & & & 494 & $100,0 \%$ & $-0,71[-0,84,-0,58]$ & 1 & \\
\hline \multicolumn{9}{|c|}{ Heterogeneidade: $\mathrm{Chi}^{2}=3,87, \mathrm{df}=3(\mathrm{P}=0,28) ; \mathrm{I}^{2}=22 \%$} & $R^{-4} L^{-4}+\frac{-1}{-2}$ & $\begin{array}{ll}0 & 1 \\
\text { Somente }\end{array}$ \\
\hline
\end{tabular}

Fig. 2 Forrest plot mostrando que a adição do procedimento tenodese extra-articular lateral à reconstrução do ligamento cruzado anterior resulta em diferença significativa do resultado funcional com base no escore Comitê Internacional de Documentação de Joelho.

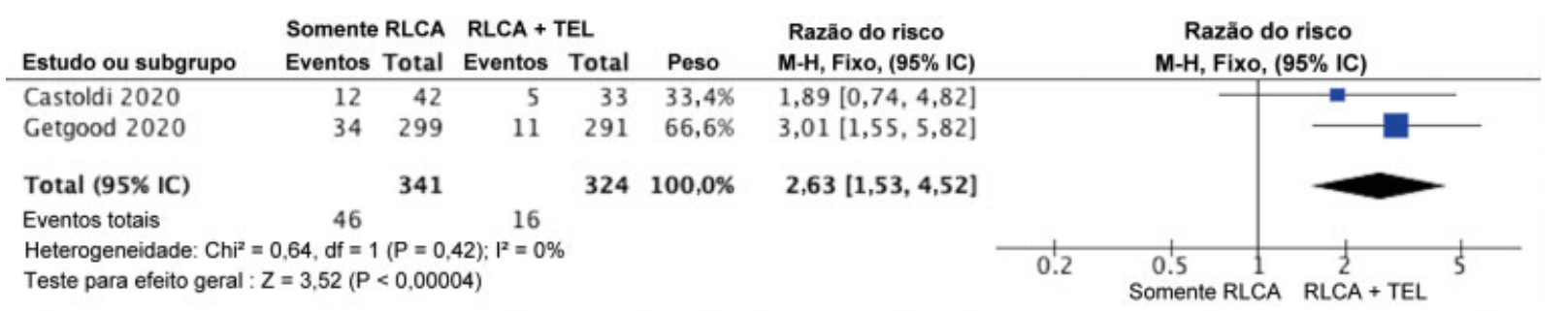

Fig. 3 Forrest Plot mostrando menor falha de enxerto no grupo reconstrução do ligamento cruzado anterior mais tenodese extra-articular lateral.

\section{Complicação}

De todas as complicações relatadas em cada estudo, a mais relatada é a falha do enxerto. ${ }^{16,19}$ A falha do enxerto foi menor no grupo RLCA mais TEL (16 dos 342 pacientes) em comparação com o grupo da RLCA simples (46 dos 341 pacientes) $(p<0,05)$. A razão de risco foi de 2,63 (IC95\%, $1,53-4,52)$.

\section{Discussão}

Esta metanálise investigou a recente evidência de alta qualidade do procedimento TEL como adição para a reconstrução da LCA. Apesar do enorme interesse atual neste procedimento, não há evidências quantitativas suficientes sobre como a adição de TEL pode afetar o resultado funcional. Portanto, este procedimento não é comum, e é indicado principalmente para revisão e mudança de pivô grau III. ${ }^{20}$ Uma revisão sistemática anterior relatou um bom resultado a médio prazo, mesmo com um número limitado de pacientes. ${ }^{7-10}$ Este estudo atual é o primeiro a apresentar análise quantitativa de estudos recentes que concluíram que o procedimento TEL em complemento à RLCA, resultou em desfecho funcional satisfatório.

A não reconstrução da estrutura anterolateral, especialmente LAL, foi considerada um dos fatores que produziram resultado insatisfatório na reconstrução anatômica do LCA. ${ }^{21}$ O LAL foi descrito por muitos autores como um componente do CAL, que foi pensado para ser um ligamento capsular médio, a camada capsulo-óssea da BI, ou uma combinação de ambos. Ele tem papel significativo na instabilidade rotatória, juntamente com a BI. ${ }^{5} \mathrm{~A}$ análise quantitativa de ensaios clínicos anteriores mostrou que a falha do enxerto é menor na reconstrução combinada do LCA com o procedimentoTEL. O procedimento adicional é benéfico na redução da complicação, provavelmente devido ao seu efeito na redução da frouxidão rotacional residual.

O procedimento de aumento anterolateral melhora significativamente a instabilidade rotativa e elimina a mudança 
de pivô em pacientes de alto risco. ${ }^{22,23}$ Ele pode ser realizado com duas técnicas: procedimento TEL e reconstrução moderna do LAL. A principal diferença entre os dois métodos é que a reconstrução do LAL permite uma reconstrução mais anatômica do que a TEL. ${ }^{24}$ Outra diferença é que os procedimentos TEL têm ponto de fixação proximal perto do epicôndilo femoral e seu ponto de fixação distal é em torno do tubérculo Gerdy, enquanto o LAL é integrado com o ligamento colateral fibular (LCF). ${ }^{4}$

Uma metanálise anterior constatou que, mesmo quando as medidas de desfecho relatadas pelo paciente foram geralmente satisfatórias, o procedimento de TEL combinado com a reconstrução da LCA deram pior estabilidade anterior. ${ }^{8}$ Por outro lado, uma metanálise relatou bons resultados de acompanhamento a médio prazo com baixas taxas de frouxidão rotatória residual, re-rupturas ou complicações. Em termos de desfecho funcional, a tendência é de que o desfecho subjetivo da IKDC seja mais semelhante entre os dois grupos no procedimento de reconstrução primária do que no procedimento de revisão. ${ }^{7}$ No entanto, os estudos incluídos em ambas as metanálises foram todos estudos retrospectivos que datam de $1986^{8}$ e $2006 .^{7}$

Este estudo concentrou-se na literatura recente com o último ensaio clínico datado de $2013,{ }^{12}$ uma vez que a técnica de artroscopia e a familiaridade do cirurgião com a técnica progrediu bem nos últimos anos. Embora existam vários ensaios clínicos comparando apenas o procedimento de reconstrução da LCA e a adição de TEL, esses estudos não encontraram diferença significativa entre os dois grupos em termos de dor e desfecho funcional, como o Índice de Simetria de Membros (LSI, na sigla em inglês). ${ }^{25-27}$ Esses estudos utilizaram diferentes técnicas e enxertos, dificultando a conclusão.

Nos últimos anos, técnicas mais uniformes foram utilizadas no procedimento TEL como complemento à RLCA com um método mais padronizado para avaliar o resultado funcional, tornando a metodologia mais rigorosa. A maioria dos estudos incluídos em nossa análise utilizou o sistema de pontuação IKDC como resultado funcional. Mesmo que Getgood et al. ${ }^{16}$ tenha relatado maior quantidade de dor nos primeiros 3 meses após a cirurgia e atraso na recuperação da força do quadríceps e redução da escala funcional da extremidade inferior (LEFS, na sigla em inglês), essas diferenças foram pequenas e transitórias.

Outro tópico interessante é como o procedimento TEL oferece benefício adicional especialmente no procedimento de RLCA tardio (após 12 meses de lesão). ${ }^{10} \mathrm{O}$ benefício adicional foi demonstrado em termos de teste de turno pivô, que não foi descrito em relação à sua classificação, podendo influenciar a análise estatística. Para fornecer uma melhor recomendação para o procedimento de RLCA tardio, estudos futuros devem considerar a utilização do resultado funcional, por exemplo, com o escore IKDC como medida de resultado. Ele é orientado para o paciente e deve ser mais considerado em estudos futuros.

Há várias limitações deste estudo que devem ser mencionadas. Em primeiro lugar, os dados disponíveis nos estudos são limitados. Por exemplo, a média e o desvio-padrão não foram bem previstos nos estudos; portanto, a análise quantitativa não pôde ser realizada. Em segundo lugar, apenas quatro dos seis estudos foram RCTs, enquanto dois deles foram estudos retrospectivos. ${ }^{12,17}$ Portanto, alguns dados apresentados na revisão sistemática não são de alta qualidade. Em terceiro lugar, todos os estudos incluídos foram publicados em inglês, o que pode resultar em um viés de publicação. Em quarto lugar, nós apenas investigamos o desfecho funcional e as complicações, uma vez que esses dois foram os fatores mais associados ao desenvolvimento da técnica de RLCA. Finalmente, a variabilidade no período de seguimento também pode influenciar nossa análise de dados.

\section{Conclusão}

Há evidências de alto nível de que o procedimento de TEL como complemento à RLCA é preferível à RLCA simples em termos de resultado funcional e taxa de falha do enxerto.

\section{Divulgação financeira \\ Sem apoio financeiro. \\ Conflito de interesses \\ Os autores não têm conflito de interesses para declarar.}

\section{Referências}

1 Getgood AMJ, Bryant DM, Litchfield R, et al. STABILITY Study Group. Lateral Extra-articular Tenodesis Reduces Failure of Hamstring Tendon Autograft Anterior Cruciate Ligament Reconstruction: 2-Year Outcomes From the STABILITY Study Randomized Clinical Trial. Am J Sports Med 2020;48(02):285-297

2 Schindler OS. Surgery for anterior cruciate ligament deficiency: a historical perspective. Knee Surg Sports Traumatol Arthrosc 2012;20(01):5-47

3 Draganich LF, Reider B, Ling M, Samuelson M. An in vitro study of an intraarticular and extraarticular reconstruction in the anterior cruciate ligament deficient knee. Am J Sports Med 1990;18(03): 262-266

4 Caterine S, Litchfield R, Johnson M, Chronik B, Getgood A. A cadaveric study of the anterolateral ligament: re-introducing the lateral capsular ligament. Knee Surg Sports Traumatol Arthrosc 2015;23(11):3186-3195

5 Musahl V, Herbst E, Burnham JM, Fu FH. The Anterolateral Complex and Anterolateral Ligament of the Knee. J Am Acad Orthop Surg 2018;26(08):261-267

6 Patel RM, Brophy RH. Anterolateral Ligament of the Knee: Anatomy, Function, Imaging, and Treatment. Am J Sports Med 2018; 46(01):217-223

7 Grassi A, Zicaro JP, Costa-Paz M, et al. ESSKA Arthroscopy Committee. Good mid-term outcomes and low rates of residual rotatory laxity, complications and failures after revision anterior cruciate ligament reconstruction (ACL) and lateral extra-articular tenodesis (LET). Knee Surg Sports Traumatol Arthrosc 2020;28 (02):418-431

8 Ra HJ, Kim JH, Lee DH. Comparative clinical outcomes of anterolateral ligament reconstruction versus lateral extra-articular tenodesis in combination with anterior cruciate ligament reconstruction: systematic review and meta-analysis. Arch Orthop Trauma Surg 2020;140(07):923-931

9 Hewison CE, Tran MN, Kaniki N, Remtulla A, Bryant D, Getgood AM. Lateral Extra-articular Tenodesis Reduces Rotational Laxity 
When Combined With Anterior Cruciate Ligament Reconstruction: A Systematic Review of the Literature. Arthroscopy 2015;31 (10):2022-2034

10 Devitt BM, Bell SW, Ardern CL, et al. The Role of Lateral Extraarticular Tenodesis in Primary Anterior Cruciate Ligament Reconstruction: A Systematic Review With Meta-analysis and Best-Evidence Synthesis. Orthop J Sports Med 2017;5(10): 2325967117731767

11 Furlan AD, Malmivaara A, Chou R, et al. Editorial Board of the Cochrane Back, Neck Group. 2015 updated method guideline for systematic reviews in the Cochrane Back and Neck Group. Spine 2015;40(21):1660-1673

12 Dejour D, Vanconcelos W, Bonin N, Saggin PRF. Comparative study between mono-bundle bone-patellar tendon-bone, double-bundle hamstring and mono-bundle bone-patellar tendon-bone combined with a modified Lemaire extra-articular procedure in anterior cruciate ligament reconstruction. Int Orthop 2013;37 (02):193-199

13 Lemaire M. Rupture ancienne du ligament croisé antérieur du genou. J Chir (Paris) 1967;93:311-320

14 Macintosh D, Darby T. Lateral Substitution Reconstruction. J Bone Joint Surg 1976;58:142

15 Christel P, Djian P. Plastie extra-articulaire antéro-latérale du genou utilisant une ténodèse courte au fascia lata. [Anteriolateral extra-articular tenodesis of the knee using a short strip of fascia lata]. Rev Chir Orthop Repar Appar Mot 2002;88(05): 508-513

16 Getgood A, Hewison C, Bryant D, et al. Stability Study Group. No Difference in Functional Outcomes When Lateral Extra-Articular Tenodesis Is Added to Anterior Cruciate Ligament Reconstruction in Young Active Patients: The Stability Study. Arthroscopy 2020; 36(06):1690-1701

17 Ferretti A, Monaco E, Ponzo A, et al. Combined Intra-articular and Extra-articular Reconstruction in Anterior Cruciate LigamentDeficient Knee: 25 Years Later. Arthroscopy 2016;32(10): 2039-2047

18 Trichine F, Alsaati M, Chouteau J, Moyen B, Bouzitouna M, Maza R. Patellar tendon autograft reconstruction of the anterior cruciate ligament with and without lateral plasty in advanced-stage chronic laxity. A clinical, prospective, randomized, single-blind study using passive dynamic X-rays. Knee 2014;21(01):58-65

19 Castoldi M, Magnussen RA, Gunst S, et al. A Randomized Controlled Trial of Bone-Patellar Tendon-Bone Anterior Cruciate Ligament Reconstruction With and Without Lateral Extra-articular Tenodesis: 19-Year Clinical and Radiological Follow-up. Am J Sports Med 2020;48(07):1665-1672

20 Tramer JS, Fidai MS, Kadri O, et al. Anterolateral Ligament Reconstruction Practice Patterns Across the United States. Orthop J Sports Med 2018;6(12):2325967118811063

21 Ferretti A, Conteduca F, Monaco E, De Carli A, D’Arrigo C. Revision anterior cruciate ligament reconstruction with doubled semitendinosus and gracilis tendons and lateral extra-articular reconstruction. J Bone Joint Surg Am 2006;88(11):2373-2379

22 Spencer L, Burkhart TA, Tran MN, et al. Biomechanical analysis of simulated clinical testing and reconstruction of the anterolateral ligament of the knee. Am J Sports Med 2015;43(09): 2189-2197

23 Ueki $\mathrm{H}$, Katagiri $\mathrm{H}$, Otabe $\mathrm{K}$, et al. Contribution of Additional Anterolateral Structure Augmentation to Controlling Pivot Shift in Anterior Cruciate Ligament Reconstruction. Am J Sports Med 2019;47(09):2093-2101

24 Slette EL, Mikula JD, Schon JM, et al. Biomechanical Results of Lateral Extra-articular Tenodesis Procedures of the Knee: A Systematic Review. Arthroscopy 2016;32(12):2592-2611

25 Noyes FR, Barber SD. The effect of an extra-articular procedure on allograft reconstructions for chronic ruptures of the anterior cruciate ligament. J Bone Joint Surg Am 1991;73(06):882-892

26 Roth JH, Kennedy JC, Lockstadt H, McCallum CL, Cunning LA. Intraarticular reconstruction of the anterior cruciate ligament with and without extra-articular supplementation by transfer of the biceps femoris tendon. J Bone Joint Surg Am 1987;69(02): 275-278

27 Oni OO, Crowder E. A comparison of isokinetics and muscle strength ratios following intra-articular and extra-articular reconstructions of the anterior cruciate ligament. Injury 1996; 27(03):195-197 